\title{
VIEWPOINT
}

\section{Insulin Signaling in Health and Disease}

\author{
Morris F. White*
}

The signaling pathways used by insulin have been identified [M. White, Insulin Signaling Pathway, Sci. STKE (Connections Map, as seen November 2003), http://stke.sciencemag. org/cgi/cm/cmp_12069]. Now our challenge is to understand how the failure of these signals is associated with obesity and the progressive failure of pancreatic beta cells that leads to diabetes. Whether better management of chronic inflammation can improve insulin action is an important area of investigation. Drugs that stimulate IRS2 (insulin receptor substrate protein 2 ) synthesis or signaling might be a good starting point. This knowledge will lead to rational strategies that prevent or cure diabetes.

$\mathbf{H}$ ow long will the patient live?" was the only question to ask until a series of discoveries beginning with Langerhans' description of pancreatic islets changed the lives of people with diabetes (1). Twenty years later, in 1889 , pancreatic secretions were shown to control blood sugar levels; however, it took another 30 years until insulin was purified from the islets before patients could ask about the "quality of their life with insulin."

For the next 50 years clinicians and scientists revealed the system-wide effects of insulin in liver, muscle, and adipose tissues, and recent work reveals insulin's effect on longevity and the central nervous system. In the 1970s, the insulin receptor was discovered, and 10 years later the demonstration of its tyrosine kinase activity pointed us toward the mechanism of signal transduction (2). Remarkably, this steady progress has not stemmed the worldwide diabetes epidemic that will take a huge toll in premature morbidity and mortality in this new century (3).

Diabetes mellitus is a complex disorder that arises from various causes, including dysregulated glucose sensing or insulin secretion (maturity-onset diabetes of the young, MODY), autoimmune-mediated $\beta$-cell destruction (type 1 ), or insufficient compensation for peripheral insulin resistance (type 2). Type 2 diabetes afflicts 18.2 million Americans. It usually occurs in middle age, but is appearing in young people owing to the close association with obesity. The signaling pathways linking obesity, peripheral insulin action, and $\beta$-cell function are important to understand.

The insulin receptor is the prototype for a family of homologous integral membrane

Howard Hughes Medical Institute, Joslin Diabetes Center, Harvard Medical School, 1 Joslin Place, Boston, MA 02215, USA

*To whom correspondence should be addressed. Email: morris.white@joslin.harvard.edu proteins composed of an extracellular insulin-binding domain that controls the activity of an intracellular tyrosine kinase. A $150-\mathrm{kb}$ gene on chromosome $19 \mathrm{com}-$ posed of 22 exons encodes the human pro-receptor. During translation, two homologous pro-receptors form a disulfidelinked dimer that is cleaved to form a tetramer of two $\alpha \beta$ dimers. Insulin binds to the extracellular $\alpha$ subunits to activate the tyrosine kinase on the intracellular portion of the transmembrane $\beta$ subunits. The activated receptor recruits and phosphorylates cellular substrates to initiate signal transduction (Fig. 1). The full insulin-signaling pathway is summarized in the STKE Connections Map (4).

Selective insulin binding is complicated by tissue-specific alternative splicing of exon-11 that directs synthesis of two insulin receptor isoforms (IRa and IRb), and by posttranslational assembly of hybrids between these isoforms and the homologous insulin-like growth factor 1 receptor (IGF1R) (5). IRb binds insulin with high affinity, whereas IRa binds insulin or IGF2 with comparable affinity. Dysregulated splicing alters fetal growth patterns and contributes to rare forms of insulin resistance in adults (6). Moreover, hybrid receptors composed of $\alpha \beta$ dimers from the IGF1R and the IRb selectively bind IGF1, whereas hybrid receptors composed of IGF1R and IRa bind IGFs and insulin with similar affinities (Fig. 1). The importance of this complexity for metabolism, growth, and survival needs to be resolved.

Evidence of a substrate for the insulin receptor tyrosine kinase came from immunoprecipitates with antibodies to phosphotyrosine that revealed a $185-\mathrm{kD}$ phosphoprotein (pp185) in insulin-stimulated hepatoma cells (7). Molecular cloning of pp185 revealed a signaling scaffold protein, and the first insulin receptor substrate protein (IRS1) (8). Most, if not all, insulin signals are produced or modulated through tyrosine phosphorylation of IRS1 or its homolog IRS2, or other scaffold proteins including IRS3, IRS4, SHC, CBL, APS and SH2B, GAB1, GAB2, DOCK1, and DOCK2. Although the role of each of these substrates merits attention, work with transgenic mice suggests that most insulin responses are mediated by IRS1 or IRS2. IRS1 controls body growth and peripheral insulin action, whereas IRS2 regulates brain growth, body weight control, glucose homeostasis, and female fertility (9). How these similar proteins mediate different physiological outcomes is an important question to answer.

IRS proteins contain an $\mathrm{NH}_{2}$-terminal pleckstrin homology $(\mathrm{PH})$ domain adjacent to a phosphotyrosine-binding (PTB) domain. The PTB domain binds directly to the phosphorylated NPXY-motif [Asn-Pro-Xaa-Tyr $\left(\mathrm{P}_{\mathrm{i}}\right)$; Xaa, any amino acid; $\mathrm{P}_{\mathrm{i}}$, inorganic phosphate] in the activated receptors for insulin, IGFs, or interleukin-4 (IL-4). The PH domain also couples IRS proteins to activated receptors, but the mechanism is unclear. Other receptors recruit and phosphorylate IRS proteins, including those for growth hormone, IL-9, IL-13 and IL-15, and various integrins. The $\mathrm{C}$-terminal tail of IRS1 and IRS2 appears to lack intrinsic catalytic activities, but contains numerous tyrosine and serine phosphorylation sites. The tyrosine phosphorylation sites bind common effector proteins, including enzymes (the phosphoinositide 3kinase, PI3K; the phosphatase SHP2; or the tyrosine kinase fyn) or adapters (SOCS1, SOCS3, GRB2, NCK, CRK, SHB, and others).

The PI3K enzyme is activated during association with IRS proteins. PI3K converts $\mathrm{PI}(4,5) \mathrm{P}_{2}$ (an inositol phospholipid in the plasma membrane) to $\mathrm{PI}(3,4,5) \mathrm{P}_{3}$ that recruits protein kinase $\mathrm{B}$ (PKB) and 3-phosphoinositidedependent protein kinase-1 (PDK1) to the plasma membrane where PDK1 activates PKB. PKB phosphorylates many substrates, including $B A D$ (important for cell survival), GSK3 $\beta$ (regulating growth and glycogen synthesis), and FOXO1 (controlling gene expression) (Fig. 1). The importance of FOXO1 was revealed by mutations in the Caenorhabditis elegans ortholog DAF16 (10). During insulin or IGF stimulation, DAF16 and FOXO1 are phosphorylated by PKB and accumulate in the cytosol. Nuclear exclusion of FOXO1 inhibits hepatic gluconeogenesis and adipocyte differentiation, whereas it promotes pancreatic $\beta$-cell function (11-13). Reduced expression of FOXO1 compensates for insulin resistance in many organisms and tissues, so drugs that sequester FOXO1 in the cytosol might be effective in treating diabetes and obesity. 
Peripheral insulin resistance contributes to type 2 diabetes, but $\beta$-cell failure is the essential feature of all types of diabetes. $\beta$ cells frequently fail to compensate for insulin resistance, apparently because the IRS2-branch of the insulin and IGF signaling cascade is also essential for $\beta$-cell growth, function, and survival (14). Increased expression of IRS2 in $\beta$ cells promotes compensatory insulin secretion in obese mice and prevents $\beta$-cell destruction induced by streptozotocin - a drug that induces type 1 diabetes; IRS2 also improves the survival and function of islet transplants in mice. Drugs that increase IRS2 synthesis might be useful treatments for diabetes. For example, expression of the IRS2 gene in $\beta$ cells is increased by agonists that stimulate adenosine $3^{\prime}, 5^{\prime}$-monophosphate (cAMP) production-including glucagon-like peptide1 or glucose itselfthrough pathways that activate the transcription factor CREB (15) (Fig. 1).

Understanding dysregulated insulin sigaling is an important goal because it causes a cohort of systemic disordersdyslipidemia, hypertension, cardiovascular disease, stroke, blindness, kidney disease, female infertility, and neurodegeneration. Subtle genetic polymorphism influences lifelong insulin sensitivity, whereas distinct monogenic disorders are difficult to identify and are usually associated with rare metabolic diseases. Acute and

chronic inflammation cause insulin resistance, which provides a framework to understand how diet, physiological stress, and obesity promote insulin resistance. Proinflammatory cytokines, including IL- 6 and tumor necrosis factor- $\alpha$ $(\mathrm{TNF}-\alpha)$ that are secreted from leukocytes during inflammation, are also produced in adipose tissue. TNF- $\alpha$ promotes serine phosphorylation of IRS1 and IRS2, which correlates closely with insulin resistance (16). Although TNF- $\alpha$ regulates various kinases, the c-Jun $\mathrm{N}$-terminal kinase (Jnk) is a prominent effector because it binds to IRS1 and IRS2 and phosphorylates serine residues that inhibit insulin-stimulated tyrosine phosphorylation (17). The knockout of
Jnk1 in obese mice, or inhibition of serine kinases by salicylates, reduces Ser phosphorylation of IRS proteins and reverses hyperglycemia, hyperinsulinemia, and dyslipidemia in obese rodents by sensitizing insulin signaling pathways (18).

Ubiquitin-mediated degradation of IRS1 and IRS2 also promotes insulin resistance (Fig. 1). IL-6 secreted from leukocytes and adipocytes increases expression of SOCS proteins, which are best known for their ability to inhibit cytokine receptor signaling. However, SOCS1 and SOCS3 also recruit an elongin $\mathrm{BC}$-based ubiquitin ligase into the

mice increases insulin sensitivity, revealing targets for inhibitor design. However, inhibition of SHIP2 or pTEN might be risky because they stabilize $\mathrm{PI}(3,4,5) \mathrm{P}_{3}$, which can stimulate cell growth. By contrast, PTP1B resides in the endoplasmic reticulum, where it dephosphorylates the insulin receptor during internalization and recycling to the plasma membrane (21). This specialized mechanism appears to limit dangerous side effects of PTP1B inhibitors.

The close association between obesity and insulin resistance, and their progression to type 2 diabetes, is a serious health problem. Whether better management of chronic inflammation can improve insulin action, promote $\beta$-cell function, and restore central nervous system appetite control is an important area of investigation. Finding drugs that stimulate IRS2 synthesis or promote its signaling might be a good starting point. However, too much insulin action might be detrimental, so future work must better resolve the network of insulin responses that are generated in various tissues, and attempt to distinguish the ones that prolong health from the ones that might diminish it.

\section{Degradation}

Gene expression

References

1. M. Bliss, The Discovery of Insulin (Univ. of Chicago Press, Chicago, IL, 1982).

2. M. Kasuga et al., Nature 298, 667 (1982).

3. P. Zimmet et al., Nature 414, 782 (2001).

4. M. White, Insulin Signaling Pathway, Sci. STKE (Connections Map, as seen November 2003), http://stke.sciencemag. org/cgi/cm/cmp_12069.

IRS-protein complexes to mediate ubiquitinylation (19). Ubiquitin-mediated degradation of IRS1 and IRS2 might be a general mechanism of cytokine-induced insulin resistance that contributes to diabetes and $\beta$-cell failure. Modern genomic approaches have revealed new cytokines secreted from adipocytes that directly influence nutrient homeostasis and insulin sensitivity, including leptin, resistin, and adiponectin. How adiponectin signaling stimulates insulin signaling deserves attention (20).

Protein or lipid phosphatases, including PTP1B, SHIP2, or PTEN, inhibit insulin signaling (Fig. 1). Disruption of each gene in
5. F. Frasca et al., Mol. Cell. Biol. 19, 3278 (1999).

6. R. S. Savkur et. al., Nature Genet. 29, 40 (2001).

7. M. F. White et al., Nature 318, 183 (1985).

8. X. J. Sun et al., Nature 352, 73 (1991).

9. M. Schubert et al., J. Neurosci. 23, 7084 (2003).

10. S. Ogg et al., Nature 389, 994 (1997).

11. J. Nakae et al., Dev. Cell 4, 119 (2003).

12. T. Kitamura et al., J. Clin. Invest 110, 1839 (2002).

13. P. Puigserver et al., Nature 423, 550 (2003).

14. A. M. Hennige et al., J. Clin. Invest. 112, 1521 (2003).

15. U. S. Jhala et al., Genes Dev. 17, 1575 (2003).

16. G. S. Hotamisligil, B. M. Spiegelman, Science 259, 87 (1999).

17. V. Aguirre et al., J. Biol. Chem. 277, 1531 (2002).

18. J. Hirosumi et al., Nature 420, 333 (2002).

19. D. L. Krebs, D. J. Hilton, Sci. STKE. 2003, E6 (2003).

20. T. Yamauchi et al., Nature 423, 762 (2003).

21. F. G. Haj, P. J. Verveer, A. Squire, B. G. Neel, P. I. Bastiaens, Science 295, 1708 (2002). 
post date 9 January 2004

VIEWPOINTS: "Insulin signaling in health and disease" by Morris F. White (5 Dec. 2003, p. 1711). In the abstract and reference (4), the link pointing to the connections map published in STKE should end with capital letters. The correct URL is http://stke.sciencemag.org/cgi/cm/CMP_12069. 